OPEN ACCESS

Edited by:

Tao Xiao,

Shenzhen University, China

Reviewed by:

Shuai Liu,

Hunan Normal University, China

Jianping Liu,

Beijing University of Chinese

Medicine, China

*Correspondence:

Xun Luo

larryluoxun@163.com

tThese authors have contributed equally to this work

Specialty section:

This article was submitted to

Cognitive Science,

a section of the journal

Frontiers in Psychology

Received: 05 April 2020

Accepted: 19 October 2020

Published: 30 November 2020

Citation:

Zhou L, Wang Y, Qiao J, Wang QM

and Luo X (2020) Acupuncture for Improving Cognitive Impairment After

Stroke: A Meta-Analysis of

Randomized Controlled Trials.

Front. Psychol. 11:549265

doi: 10.3389/fpsyg.2020.549265

\section{Acupuncture for Improving Cognitive Impairment After Stroke: A Meta-Analysis of Randomized Controlled Trials}

\author{
Liang Zhou ${ }^{1+}$, Yao Wang ${ }^{2 \dagger}$, Jun Qiao ${ }^{3}$, Qing Mei Wang ${ }^{4}$ and Xun Luo ${ }^{2,5 *}$ \\ ${ }^{1}$ Department of Reproductive Medicine, Peking University Shenzhen Hospital, Shenzhen, China, ${ }^{2}$ Department of \\ Rehabilitation Medicine, Dapeng New District Nan'ao People's Hospital, Shenzhen, China, ${ }^{3}$ The Second Rehabilitation \\ Hospital of Shanghai, Shanghai, China, ${ }^{4}$ Stroke Biological Recovery Laboratory, Spaulding Rehabilitation Hospital, The \\ Teaching Affiliate of Harvard Medical School, Boston, MA, United States, ${ }^{5}$ Kerry Rehabilitation Medicine Research Institute, \\ Shenzhen, China
}

Objective: This meta-analysis evaluated the efficacy of acupuncture in improving cognitive impairment of post-stroke patients.

Design: Randomized controlled trials (RCTs) investigating the effects of acupuncture compared with no treatment or sham acupuncture on post-stroke cognitive impairment (PSCI) before December 2019 were identified from databases (PubMed, EMBASE, Ovid library, Cochrane Library, Chinese National Knowledge Infrastructure, VIP Chinese Periodical Database, Wanfang Database, and SinoMed). The literature searching and data extracting were independently performed by two investigators. Study quality was assessed using the Cochrane Handbook for Systematic Reviews of Interventions. Meta-analyses were performed for the eligible RCTs with Revman 5.3 software.

Results: Thirty-seven RCTs (2,869 patients) were included in this meta-analysis. Merged Random-effects estimates of the gain of MMSE (Mini-Mental State Examination) or MoCA (Montreal Cognitive Assessment) were calculated for the comparison of acupuncture with no acupuncture or sham acupuncture. Following 2-8 weeks of intervention with acupuncture, pooled results demonstrated significant effects of acupuncture in improving $\mathrm{PSCl}$ assessed by MMSE (MD [95\% Cl] = 2.88 [2.09, 3.66], $p<0.00001)$ or MoCA (MD $[95 \% \mathrm{Cl}]=2.66[1.95,3.37], p<0.00001)$.

Conclusion: The results suggest that acupuncture was effective in improving PSCl and supported the needs of more rigorous design with large-scale randomized clinical trials to determine its therapeutic benefits.

Keywords: MMSE, cognitive impairment, electroacupuncture, acupuncture, post stroke

\section{INTRODUCTION}

Stroke is a disease that causes high rates of mortality and disability worldwide (Wu et al., 2010). Cognitive impairment is a frequent condition after stroke (Tatemichi et al., 1994; Patel et al., 2003), and its prevalence ranges from 17 to 92\% (Pasi et al., 2012). Cognitive rehabilitation could enhance the quality of life for post-stroke patients, which included a 
comprehensive cognitive improvement program treating cognitive dysfunction involving disorientation, sensory disorders, attention disorders, executive function disorders, and memory disorders (Berrol, 1990; Choi and Twamley, 2013). The clinical depression is characterized by behavioral, cognitive, and emotional features (Merriman et al., 2019). Cognitive performance is associated with symptoms of depression (Nakling et al., 2017), and early cognitive impairment after stroke predicts long-term depressive symptoms in patients (Nys et al., 2006).

Acupuncture therapy has been used widely to promote motor recovery after stroke (Hu et al., 1993; Lee et al., 2003). Because of its low cost with low adverse events, acupuncture has also been used to improve the cognitive function of stroke patients, mostly in China, and it is receiving increasing attention among western countries (Johansson et al., 1993; NIH consensus conference, 1998). A considerable number of clinical trials showed the potential role of acupuncture as a promising treatment for post-stroke cognition impairment, but some trials suggest that acupuncture does not affect post-stroke cognitive impairment (PSCI) (Guo et al., 2007). The conflicting results may be caused by a small sample size of the trials and a flaw of study design.

Two systematic reviews (Liu et al., 2014; Wang, 2017) were performed; however, the studies were limited by small sample size. Liu et al. (2014) reported the meta-analysis results of 21 trials from 2006 to 2012; however, those trials had 12 different methods to evaluate cognitive function. Therefore, the largest dataset had only 116 patients from four studies [with MiniMental State Examination (MMSE) as the outcome measure]. In Wang's systematic review, only 15 studies with 1,085 subjects from 2008 to 2016 were included.

Since the last systematic review, many more clinical trials of acupuncture for post-stroke impairment were conducted; however, all of these clinical trials were limited by a small sample size or inconsistent selection criteria for the assessment of cognitive function. Therefore, with the further increased randomized controlled trials (RCT) evidence, there is a strong need to perform a systematic review to evaluate the therapeutic effect of acupuncture to treat PSCI.

In this study, we hypothesize that acupuncture is effective to improve cognitive function after stroke as compared to sham or no acupuncture. This systematic review and meta-analysis aimed to validate the efficacy of acupuncture in treatment for PSCI with MMSE or Montreal Cognitive Assessment (MoCA), which are the most generally used assessment tools for cognitive impairment (Foreman et al., 1996; Nasreddine et al., 2005).

\section{MATERIALS AND METHODS}

\section{Inclusion Criteria and Exclusion Criteria}

The inclusion criteria were the following: (1) type of studies: only randomized controlled trials (RCTs) of acupuncture for PSCI in English or Chinese language which were published before December 2019 were included; (2) type of participants: poststroke patients (over 18 years old) with PSCI were included without restriction on gender, race, or nation; (3) type of interventions: the RCTs that used traditional acupuncture or electroacupuncture to treat PSCI were included; (4) outcome measurements: the outcome was assessed by MMSE or MoCA; and (5) type of comparators: the comparative interventions could be sham acupuncture or conventional treatment with rehabilitation. A RCT was included if acupuncture was used at acupoints as the sole treatment or as an adjunct to other treatments for cognition impairment after stroke. If studies included three or over three groups with only one group receiving acupuncture, and there is a control group without receiving acupuncture treated consistent with the acupuncture group, the data of acupuncture group, and control group were chosen for this study. If studies included three or over three groups with two or over two groups receiving acupuncture, a routine acupuncture group was chosen as the experiment group, and the group not receiving any acupuncture treated consistent with the acupuncture group was chosen as a control group.

The exclusion criteria were the following: (1) cognition impairment caused by other diseases except for stroke; (2) studies without a control group (control group treated consistent with the acupuncture group except receiving acupuncture); (3) studies compared different types of acupuncture; (4) studies compared the effect of acupuncture with a drug; (5) studies adopted complex treatment without specifying the sole effects of acupuncture; (6) cognition outcome measured by another assessing system except for MMSE or MoCA; (7) studies without standardized indices of curative effect or detailed results of treatment will be excluded; and (8) full texts cannot be obtained or the data cannot be extracted.

\section{Identification of Eligible Trials}

For search strategy, we searched articles published before December 2019 in the following databases: Chinese Science and Technology Periodical Database (VIP), China National Knowledge Infrastructure (CNKI), Wan Fang Database, PubMed, Embase, Web of Science, and the Ovid Library, and using the combining medical subject headings and keyword terms for stroke, acupuncture, and cognition. The search terms included "acupuncture/electroacupuncture" AND "stroke/stroke rehabilitation/cerebrovascular accident/brain ischemia/cerebral hemorrhage/CVA/cerebral embolism" AND "cognition/cognitive." At the same time, some studies were extracted from the references in the full-text articles. Articles were restricted to English and Chinese languages.

\section{Assessment of Risk of Bias}

The methodological quality and the risk of bias of the included studies were compiled using the risk of bias tool in the Cochrane Handbook for Systematic Reviews of Interventions (version 5.3) by two reviewers (L.Z. and Y.W.) independently. This instrument included seven specific domains: random sequence generation, allocation concealment, blinding of participants and personnel, blinding of outcome assessment, incomplete outcome data, selective reporting, and other bias.

\section{Data Extraction}

Studies were screened by two investigators independently. Disagreements were settled by consensus or a third investigator. The extracted data included general characteristics (author and 
year of publication), patient characteristics (sample size, mean age, and disease type), intervention characteristics (type and duration), and main outcomes and adverse events. When a given study reported the outcome with more than one cognitive function assessment, we gave preference primarily to MMSE or MoCA.

\section{Statistical Analysis}

All statistical analyses were performed with Revman 5.3 software (The Cochrane Collaboration software unpdate). Since the outcomes in studies were continuous variables, the mean differences (MDs) with 95\% confidence intervals (CIs) were calculated. Heterogeneity was showed by $I^{2}$ index values with a $p$-value and percentage, respectively. A fixed-effects model would be used in a meta-analysis when heterogeneity was adopted $\left(I^{2}<\right.$ $25 \%$ or $50 \%>I^{2} \geq 25 \%$ with $\left.p>0.1\right)$. Otherwise $\left(I^{2} \geq 50 \%\right.$ or $50 \%>I^{2} \geq 25 \%$ with $\left.p \leq 0.1\right)$ the random-effects model would be used.

The stability of the results was confirmed by sensitivity analysis. Publication bias was assessed by Begg's test with STATA software (version 12.0, Stata Corp). Quality of evidence was assessed with GRADEpro in website (www.gradepro.org).

\section{RESULTS}

\section{Eligible Studies}

The workflow of literature screening and inclusion is shown in Figure 1. The initial literature search yielded 977 studies. Out of 977 studies, 72 studies were duplicated. A total of 905 studies were assessed for eligibility by titles and abstracts screening. There were 69 papers that compared the effect of acupuncture in patients with PSCI. With full-text reading, 32 articles were excluded, and 37 studies were included in the synthesis. The 37 studies are 31 journal articles (Huang et al., 2008; Li and Zhang, 2008; Lin et al., 2010; Jia and Meng, 2011; Sun and Wu, 2011; Bai et al., 2012; Li et al., 2012, 2019; Liu and Feng, 2013; Song et al., 2013; Wang, 2014, 2019; Wang et al., 2014, 2019; Yang, 2014; Liu et al., 2015a,b; Zeng et al., 2015; Cai et al., 2016; Shao, 2016; Liu, 2017; Wang H. et al., 2017; Wang Z. et al., 2017; Zhang et al., 2017; Du et al., 2018; Jia and Lv, 2018; Ma et al., 2018; Wang and Li, 2018; Shi and Wei, 2019; Zhou H. et al., 2019; Zhou J. et al., 2019) and 6 dissertations (Jiang, 2011; Kang, 2011; Yang, 2011; Feng, 2013; Lu, 2014; Sun, 2017), which involved 2,869 patients (1,442 patients in the treatment group and 1,427 patients in the control group) in total. All those studies were conducted in China. Thirty-six papers were published in the Chinese language. Table 1 shows the detailed information on the characteristics of the included studies. The ages of the patients range from 35 to 80 years. Seven trials did not describe the sex of the patients, while other trials included more male than female participants. The treatment period ranged from 2 to 12 weeks; the frequency of the sessions ranged from two sessions per day to five sessions per week. The chronicity of stroke ranged from 3 to 1,080 days, but most of those patients were treated within 6 months of onset. Twenty-three trials were conducted by manual acupuncture stimulation, and the other 14 trials used electroacupuncture only.
The cognitive function assessment of all included studies was MMSE or MoCA.

\section{Assessment of Risk of Bias}

All RCTs had a low risk of bias (ROB) about adequate sequence generation. Eight RCTs had a low ROB with allocation concealment, while 9 RCTs had a high ROB, and 20 had an unclear ROB. Concerning participant blinding, one RCT had low $\mathrm{ROB}$ and the others had a high or unclear ROB. About assessor blinding, only three RCTs had a low ROB.

All 37 RCTs had a low ROB in incomplete outcome data addressed and selective outcome reporting. Thirty-four RCTs had an unclear ROB in other sources of bias. The results of the ROB assessment are shown in Table 2, Figure 2.

\section{Meta-Analysis of the Results}

The pooled meta-analysis of the data showed a weighted mean difference of 2.88 and $95 \%$ confidence intervals (CI) of 2.09-3.66 on the MMES ( $p<0.001, n=2,349$; Figure 3).

Subgroup analyses showed weighted mean differences of 2.52 (95\% CI: $1.86-3.18, n=1,622$ ) and 3.45 (95\% CI: 2.09$3.66, n=727)$ for acupuncture subgroup and electropuncture subgroup, respectively.

The pooled meta-analysis of the data showed a weighted mean difference of 2.66 and $95 \%$ confidence intervals of $1.95-3.37$ on the MoCA ( $p<0.001, n=1,129$; Figure 4).

Subgroup analyses showed weighted mean differences of 2.55 (95\% CI: $1.71-3.39, n=652)$ and 2.81 (95\% CI: $1.42-$ 4.02, $n=477)$ for acupuncture subgroup and electropuncture subgroup, respectively.

The results indicated that acupuncture had a significant effect on PSCI, and no adverse events were reported in those studies.

\section{Sensitivity Analysis, Publication Bias, and Overall Quality of Evidence}

High heterogeneity was shown in results $\left(I^{2}=93\right.$ and 55 for MMSE and MoCA, respectively), so subgroup analyses were done based on different methods of acupuncture between manual acupuncture (acupuncture) and electropuncture. The results of subgroup analyses showed that intra-group heterogeneity remained high in subgroups $\left(I^{2}>50\right)$, and the inter-group heterogeneity between subgroups was not too much $\left(I^{2}<\right.$ 50). Then sensitivity analysis was conducted by excluding the maximum weight studies in outcomes on subgroup analyses. The results showed that there was little influence on the pooled $\mathrm{MD}$ value. Then a study was removed at a time and the others analyzed to assess whether the results could have been influenced significantly by a single study. The results also showed no apparent fluctuation. These analyses confirmed the stability of the results of pooled MD value. Begg's tests showed no significant publication bias with symmetrical funnel plots. The overall quality of evidence was rated as moderate for MMSE and MoCA (Figure 5). 


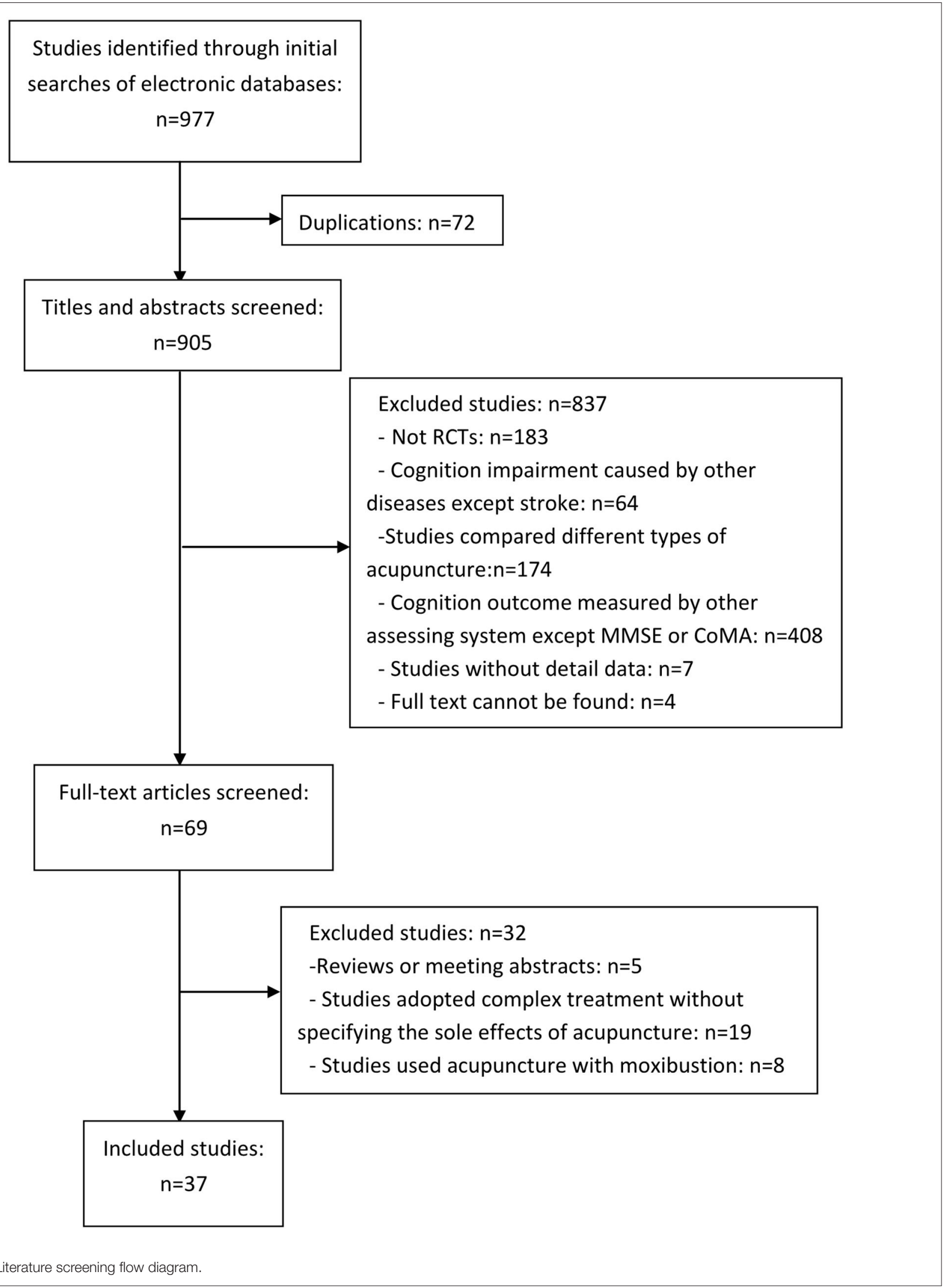




\begin{tabular}{|c|c|c|c|c|c|c|c|c|c|c|c|}
\hline \multirow[t]{2}{*}{ References } & \multicolumn{2}{|c|}{ Patients no. } & \multicolumn{2}{|c|}{ Ages (years) } & \multirow[t]{2}{*}{ Type of stroke } & \multirow{2}{*}{$\begin{array}{l}\text { Outcome } \\
\text { measures } \\
\text { (MMSE/MoCA) }\end{array}$} & \multirow{2}{*}{$\begin{array}{l}\text { Therapy } \\
\text { duration } \\
\text { (wk) }\end{array}$} & \multicolumn{2}{|c|}{ Intervation } & \multirow{2}{*}{$\begin{array}{l}\text { Source of diagnostic } \\
\text { criteria for cerebral } \\
\text { vascular diseases }\end{array}$} & \multirow{2}{*}{$\begin{array}{l}\text { Source of } \\
\text { diagnostic } \\
\text { criteria for PSCI }\end{array}$} \\
\hline & Treatmen & ontrol & Treatment & Control & & & & Treatment & Control & & \\
\hline Sun (2017) & 30 & 28 & $60.63 \pm 8.273$ & $61.29 \pm 7.693$ & $\begin{array}{l}\text { Ischemic stroke or } \\
\text { hemorrhage }\end{array}$ & MMSE and MoCA & 6 & $\begin{array}{l}\text { Acupuncture+ } \\
\text { control treatment }\end{array}$ & $\begin{array}{l}\text { Conventional } \\
\text { treatment }+ \\
\text { rehabilitation }\end{array}$ & $\begin{array}{l}\text { FNACCVD confirmed } \\
\text { by head CT or MRI }\end{array}$ & DSM-IV \\
\hline Shao (2016) & 28 & 28 & & \pm 5 & $\begin{array}{l}\text { Ischemic stroke or } \\
\text { hemorrhage }\end{array}$ & MMSE & 12 & $\begin{array}{l}\text { Acupuncture+ } \\
\text { control treatment }\end{array}$ & $\begin{array}{l}\text { Conventional } \\
\text { treatment }+ \\
\text { rehabilitation }\end{array}$ & $\begin{array}{l}\text { CECS, Chinese expert } \\
\text { consensus standards }\end{array}$ & DSM-IV \\
\hline Liu et al. (2015a) & 32 & 30 & $51.97 \pm 9.11$ & $51.30 \pm 10.57$ & $\begin{array}{l}\text { Ischemic stroke or } \\
\text { hemorrhage }\end{array}$ & MoCA & 4 & $\begin{array}{l}\text { Electropuncture+ } \\
\text { control treatment }\end{array}$ & $\begin{array}{l}\text { Conventional } \\
\text { treatment }+ \\
\text { rehabilitation }\end{array}$ & $\begin{array}{l}\text { CECS, Chinese expert } \\
\text { consensus standards }\end{array}$ & MoCA \\
\hline Cai et al. (2016) & 52 & 49 & $57.75 \pm 13.74$ & $56.18 \pm 11.86$ & $\begin{array}{l}\text { Ischemic stroke or } \\
\text { hemorrhage }\end{array}$ & MMSE and MoCA & 12 & $\begin{array}{l}\text { Acupuncture+ } \\
\text { control treatment }\end{array}$ & $\begin{array}{l}\text { Conventional } \\
\text { treatment }+ \\
\text { rehabilitation }\end{array}$ & $\begin{array}{l}\text { CECS, Chinese expert } \\
\text { consensus standards }\end{array}$ & MMSE \\
\hline Zeng et al. (2015) & 50 & 50 & $66 \pm 12$ & $68 \pm 10$ & $\begin{array}{l}\text { Ischemic stroke or } \\
\text { hemorrhage }\end{array}$ & MoCA & $4 / 8$ & $\begin{array}{l}\text { Electropuncture+ } \\
\text { control treatment }\end{array}$ & $\begin{array}{l}\text { Conventional } \\
\text { treatment }+ \\
\text { rehabilitation }\end{array}$ & $\begin{array}{l}\text { FNACCVD confirmed } \\
\text { by head CT or MRI }\end{array}$ & MoCA \\
\hline Wang (2014) & 33 & 31 & 66.4 & \pm 3.0 & $\begin{array}{l}\text { Ischemic stroke or } \\
\text { hemorrhage }\end{array}$ & MMSE & 3 & $\begin{array}{l}\text { Acupuncture+ } \\
\text { control treatment }\end{array}$ & $\begin{array}{l}\text { Conventional } \\
\text { treatment }+ \\
\text { xingnaojing }\end{array}$ & $\begin{array}{l}\text { FNACCVD confirmed } \\
\text { by head CT or MRI }\end{array}$ & Not shown \\
\hline Lu (2014) & 30 & 30 & $63.27 \pm 11.88$ & $63.90 \pm 8.48$ & $\begin{array}{l}\text { Ischemic stroke or } \\
\text { hemorrhage }\end{array}$ & MoCA & 4 & $\begin{array}{l}\text { Acupuncture+ } \\
\text { control treatment }\end{array}$ & $\begin{array}{l}\text { Conventional } \\
\text { treatment }+ \\
\text { rehabilitation }\end{array}$ & $\begin{array}{l}\text { CECS, Chinese expert } \\
\text { consensus standards }\end{array}$ & MoCA \\
\hline Zhai (2012) & 55 & 55 & & 99.2 & Ischemic stroke & MMSE & 12 & $\begin{array}{l}\text { Acupuncture+ } \\
\text { control treatment }\end{array}$ & $\begin{array}{l}\text { Conventional } \\
\text { treatment }+ \\
\text { rehabilitation }\end{array}$ & $\begin{array}{l}\text { FNACCVD confirmed } \\
\text { by head CT or MRI }\end{array}$ & Not shown \\
\hline Bai et al. (2012) & 30 & 30 & $60 \pm 6$ & $60 \pm 6$ & $\begin{array}{l}\text { Ischemic stroke or } \\
\text { hemorrhage }\end{array}$ & MMSE & 4 & $\begin{array}{l}\text { Acupuncture+ } \\
\text { control treatment }\end{array}$ & $\begin{array}{l}\text { Conventional } \\
\text { treatment + } \\
\text { piracetam }\end{array}$ & $\begin{array}{l}\text { FNACCVD confirmed } \\
\text { by head CT or MRI }\end{array}$ & CCSE \\
\hline Li et al. (2012) & 48 & 46 & $68.29 \pm 8.22$ & $69.22 \pm 7.88$ & $\begin{array}{l}\text { Ischemic stroke or } \\
\text { hemorrhage }\end{array}$ & MMSE & 12 & $\begin{array}{l}\text { Acupuncture+ } \\
\text { control treatment }\end{array}$ & $\begin{array}{l}\text { Conventional } \\
\text { treatment }+ \\
\text { nimodipine }\end{array}$ & $\begin{array}{l}\text { FNACCVD confirmed } \\
\text { by head CT or MRI }\end{array}$ & MMSE \\
\hline Yang (2011) & 20 & 20 & $59.00 \pm 8.46$ & $59.30 \pm 8.42$ & $\begin{array}{l}\text { Ischemic stroke or } \\
\text { hemorrhage }\end{array}$ & MMSE & 8 & $\begin{array}{l}\text { Acupuncture+ } \\
\text { control treatment }\end{array}$ & $\begin{array}{l}\text { Conventional } \\
\text { treatment }+ \\
\text { rehabilitation }\end{array}$ & $\begin{array}{l}\text { FNACCVD confirmed } \\
\text { by head CT or MRI }\end{array}$ & MMSE \\
\hline $\begin{array}{l}\text { Sun and Wu } \\
\text { (2011) }\end{array}$ & 36 & 36 & $63.6 \pm 5.8$ & $64.1 \pm 5.5$ & Ischemic stroke & MMSE & 4 & $\begin{array}{l}\text { Acupuncture+ } \\
\text { control treatment }\end{array}$ & $\begin{array}{l}\text { Conventional } \\
\text { treatment + } \\
\text { aricept }\end{array}$ & $\begin{array}{l}\text { FNACCVD confirmed } \\
\text { by head CT or MRI }\end{array}$ & MMSE \\
\hline Kang (2011) & 24 & 24 & $60.67 \pm 6.93$ & $62.71 \pm 5.34$ & $\begin{array}{l}\text { Ischemic stroke or } \\
\text { hemorrhage }\end{array}$ & MMSE & 8 & $\begin{array}{l}\text { Electropuncture+ } \\
\text { control treatment }\end{array}$ & $\begin{array}{l}\text { Conventional } \\
\text { treatment }+ \\
\text { rehabilitation }\end{array}$ & $\begin{array}{l}\text { FNACCVD confirmed } \\
\text { by head CT or MRI }\end{array}$ & MMSE \\
\hline Jiang (2011) & 20 & 20 & $62.85 \pm 5.67$ & $61.75 \pm 6.35$ & $\begin{array}{l}\text { Ischemic stroke or } \\
\text { hemorrhage }\end{array}$ & MMSE & 8 & $\begin{array}{l}\text { Electropuncture+ } \\
\text { control treatment }\end{array}$ & $\begin{array}{l}\text { Conventional } \\
\text { treatment }+ \\
\text { rehabilitation }\end{array}$ & $\begin{array}{l}\text { FNACCVD confirmed } \\
\text { by head CT or MRI }\end{array}$ & CCSE \\
\hline
\end{tabular}


TABLE 1 | Continued

\begin{tabular}{|c|c|c|c|c|c|c|c|c|c|c|c|}
\hline \multirow[t]{2}{*}{ References } & \multirow{2}{*}{\multicolumn{2}{|c|}{$\begin{array}{c}\text { Patients no. } \\
\text { Treatment Control }\end{array}$}} & \multicolumn{2}{|c|}{ Ages (years) } & \multirow[t]{2}{*}{ Type of stroke } & \multirow{2}{*}{$\begin{array}{l}\text { Outcome } \\
\text { measures } \\
\text { (MMSE/MoCA) }\end{array}$} & \multirow{2}{*}{$\begin{array}{l}\text { Therapy } \\
\text { duration } \\
\text { (wk) }\end{array}$} & \multicolumn{2}{|c|}{ Intervation } & \multirow{2}{*}{$\begin{array}{l}\text { Source of diagnostic } \\
\text { criteria for cerebral } \\
\text { vascular diseases }\end{array}$} & \multirow{2}{*}{$\begin{array}{l}\text { Source of } \\
\text { diagnostic } \\
\text { criteria for PSCI }\end{array}$} \\
\hline & & & Treatment & Control & & & & Treatment & Control & & \\
\hline $\begin{array}{l}\text { Jia and Meng } \\
\text { (2011) }\end{array}$ & 50 & 50 & $65 \pm 2$ & $58 \pm 3$ & Ischemic stroke & $\mathrm{MoCA}$ & 12 & $\begin{array}{l}\text { Acupuncture+ } \\
\text { control treatment }\end{array}$ & $\begin{array}{l}\text { Conventional } \\
\text { treatment }+ \\
\text { rehabilitation + } \\
\text { nimodipine }\end{array}$ & $\begin{array}{l}\text { CECS, Chinese expert } \\
\text { consensus standards }\end{array}$ & $\begin{array}{l}\text { Diagnosis criteria } \\
\text { shown in reference } \\
\text { (Jia, 2004) }\end{array}$ \\
\hline Lin et al. (2010) & 30 & 30 & $63 \pm 17$ & $56 \pm 13$ & Ischemic stroke & MMSE & 3 & $\begin{array}{l}\text { Acupuncture+ } \\
\text { control treatment }\end{array}$ & $\begin{array}{l}\text { Conventional } \\
\text { treatment }+ \\
\text { xingnaojing }\end{array}$ & $\begin{array}{l}\text { FNACCVD confirmed } \\
\text { by head CT or MRI }\end{array}$ & MMSE \\
\hline Huang et al. (2008) & 40 & 40 & $59.22 \pm 10.6$ & $61.05 \pm 9.68$ & Ischemic stroke & MMSE & 4 & $\begin{array}{l}\text { Acupuncture+ } \\
\text { control treatment }\end{array}$ & $\begin{array}{l}\text { Conventional } \\
\text { treatment }+ \\
\text { xingnaojing }\end{array}$ & $\begin{array}{l}\text { FNACCVD confirmed } \\
\text { by head CT or MRI }\end{array}$ & CECVCl \\
\hline Shi and Wei (2019) & 55 & 55 & $60.31 \pm 2.73$ & $60.24 \pm 2.65$ & Stroke & MMSE & 4 & $\begin{array}{l}\text { Acupuncture+ } \\
\text { control treatment }\end{array}$ & $\begin{array}{l}\text { Conventional } \\
\text { treatment }+ \\
\text { rehabilitation }\end{array}$ & Not shown & Not shown \\
\hline $\begin{array}{l}\text { Zhou J. et al. } \\
\text { (2019) }\end{array}$ & 60 & 60 & $61.44 \pm 8.77$ & $62.04 \pm 8.69$ & $\begin{array}{l}\text { Ischemic stroke or } \\
\text { hemorrhage }\end{array}$ & MMSE and MoCA & 4 & $\begin{array}{l}\text { Electropuncture+ } \\
\text { control treatment }\end{array}$ & $\begin{array}{l}\text { Conventional } \\
\text { treatment }+ \\
\text { rehabilitation }\end{array}$ & $\begin{array}{l}\text { CECS, Chinese expert } \\
\text { consensus standards }\end{array}$ & DSM \\
\hline Feng (2013) & 40 & 40 & $51.65 \pm 12.47$ & $52.13 \pm 12.77$ & $\begin{array}{l}\text { Ischemic stroke or } \\
\text { hemorrhage }\end{array}$ & MMSE and MoCA & 4 & $\begin{array}{l}\text { Electropuncture+ } \\
\text { control treatment }\end{array}$ & $\begin{array}{l}\text { Conventional } \\
\text { treatment }+ \\
\text { rehabilitation }\end{array}$ & $\begin{array}{l}\text { FNACCVD confirmed } \\
\text { by head CT or MRI }\end{array}$ & DSM-IV \\
\hline Wang et al. (2019) & 59 & 59 & $68.88 \pm 3.64$ & $67.71 \pm 3.02$ & Ischemic stroke & MMSE & 4 & $\begin{array}{l}\text { Acupuncture+ } \\
\text { control treatment }\end{array}$ & $\begin{array}{l}\text { Conventional } \\
\text { treatment }+ \\
\text { Atorvastatin }\end{array}$ & $\begin{array}{l}\text { FNACCVD confirmed } \\
\text { by head CT or MRI }\end{array}$ & $\begin{array}{l}\text { There are } \\
\text { symptoms such as } \\
\text { memory loss }\end{array}$ \\
\hline Li et al. (2019) & 40 & 40 & $66.9 \pm 5.9$ & $67.4 \pm 6.1$ & $\begin{array}{l}\text { Ischemic stroke or } \\
\text { hemorrhage }\end{array}$ & MMSE and MoCA & $6 / 12$ & $\begin{array}{l}\text { Acupuncture+ } \\
\text { control treatment }\end{array}$ & $\begin{array}{l}\text { Conventional } \\
\text { treatment+ } \\
\text { Donepezil }\end{array}$ & $\begin{array}{l}\text { FNACCVD confirmed } \\
\text { by head CT or MRI }\end{array}$ & DSM-IV-R \\
\hline Zhang et al. (2017) & 42 & 42 & $62.28 \pm 10.68$ & $63.07 \pm 10.59$ & Stroke & MMSE & 4 & $\begin{array}{l}\text { Acupuncture+ } \\
\text { control treatment }\end{array}$ & $\begin{array}{l}\text { Conventional } \\
\text { treatment }+ \\
\text { rehabilitation }+ \\
\text { Atorvastatin }\end{array}$ & $\begin{array}{l}\text { FNACCVD confirmed } \\
\text { by head CT or MRI }\end{array}$ & $\begin{array}{l}\text { There are } \\
\text { symptoms such as } \\
\text { memory loss }\end{array}$ \\
\hline $\begin{array}{l}\text { Wang H. et al. } \\
\text { (2017) }\end{array}$ & 30 & 30 & $53.27 \pm 11.62$ & $56.73 \pm 9.31$ & $\begin{array}{l}\text { Ischemic stroke or } \\
\text { hemorrhage }\end{array}$ & MMSE & 8 & $\begin{array}{l}\text { Electropuncture+ } \\
\text { control treatment }\end{array}$ & $\begin{array}{l}\text { Conventional } \\
\text { treatment }+ \\
\text { rehabilitation }\end{array}$ & $\begin{array}{l}\text { FNACCVD confirmed } \\
\text { by head CT or MRI }\end{array}$ & DSM-IV \\
\hline $\begin{array}{l}\text { Zhou H. et al. } \\
\text { (2019) }\end{array}$ & 40 & 40 & $61.5 \pm 5.7$ & $61.5 \pm 4.4$ & Ischemic stroke & MMSE and MoCA & 6 & $\begin{array}{l}\text { Electropuncture+ } \\
\text { control treatment }\end{array}$ & $\begin{array}{l}\text { Conventional } \\
\text { treatment }+ \\
\text { rehabilitation + } \\
\text { Perindopril }\end{array}$ & $\begin{array}{l}\text { FNACCVD confirmed } \\
\text { by head CT or MRI }\end{array}$ & Not shown \\
\hline $\begin{array}{l}\text { Wang Z. et al. } \\
\text { (2017) }\end{array}$ & 30 & 30 & $61.13 \pm 11.42$ & $60.06 \pm 11.17$ & $\begin{array}{l}\text { Ischemic stroke or } \\
\text { hemorrhage }\end{array}$ & MMSE and MoCA & 8 & $\begin{array}{l}\text { Acupuncture+ } \\
\text { control treatment }\end{array}$ & $\begin{array}{l}\text { Conventional } \\
\text { treatment + } \\
\text { rehabilitation }\end{array}$ & $\begin{array}{l}\text { FNACCVD confirmed } \\
\text { by head CT or MRI }\end{array}$ & DSM-IV-R \\
\hline
\end{tabular}


TABLE 1 | Continued

\begin{tabular}{|c|c|c|c|c|c|c|c|c|c|c|c|}
\hline \multirow[t]{2}{*}{ References } & \multicolumn{2}{|c|}{ Patients no. } & \multicolumn{2}{|c|}{ Ages (years) } & \multirow[t]{2}{*}{ Type of stroke } & \multirow{2}{*}{$\begin{array}{l}\text { Outcome } \\
\text { measures } \\
\text { (MMSE/MoCA) }\end{array}$} & \multirow{2}{*}{$\begin{array}{l}\text { Therapy } \\
\text { duration } \\
\text { (wk) }\end{array}$} & \multicolumn{2}{|c|}{ Intervation } & \multirow{2}{*}{$\begin{array}{l}\text { Source of diagnostic } \\
\text { criteria for cerebral } \\
\text { vascular diseases }\end{array}$} & \multirow{2}{*}{$\begin{array}{l}\text { Source of } \\
\text { diagnostic } \\
\text { criteria for PSCI }\end{array}$} \\
\hline & Treatme & Control & Treatment & Control & & & & Treatment & Control & & \\
\hline Wang et al. (2019) & 78 & 78 & $69.04 \pm 3.48$ & $68.92 \pm 3.65$ & stroke & MMSE & 4 & $\begin{array}{l}\text { acupuncture+ } \\
\text { control treatment }\end{array}$ & $\begin{array}{l}\text { Conventional } \\
\text { treatment }+ \\
\text { Tongluofuzheng } \\
\text { decoction }\end{array}$ & Not shown & Not shown \\
\hline $\begin{array}{l}\text { Wang and Li } \\
\text { (2018) }\end{array}$ & 64 & 64 & $71.42 \pm 8.67$ & $69.33 \pm 7.56$ & Ischemic stroke & MMSE and MoCA & $6 / 10$ & $\begin{array}{l}\text { Acupuncture+ } \\
\text { control treatment }\end{array}$ & $\begin{array}{l}\text { Conventional } \\
\text { treatment }+ \\
\text { rehabilitation }+ \\
\text { nimodipine }\end{array}$ & $\begin{array}{l}\text { CECS, Chinese expert } \\
\text { consensus standards }\end{array}$ & $\begin{array}{l}\text { Diagnosis criteria } \\
\text { shown in reference } \\
\text { (Zhang and Wang, } \\
\text { 2004) }\end{array}$ \\
\hline Yang (2014) & 40 & 40 & \multicolumn{2}{|c|}{$61.7 \pm 4.8$} & Stroke & MMSE & 3 & $\begin{array}{l}\text { Acupuncture+ } \\
\text { control treatment }\end{array}$ & $\begin{array}{l}\text { Conventional } \\
\text { treatment }+ \\
\text { xingnaojing }\end{array}$ & $\begin{array}{l}\text { FNACCVD confirmed } \\
\text { by head CT or MRI }\end{array}$ & Not shown \\
\hline Ma et al. (2018) & 30 & 30 & $60.97 \pm 7.15$ & $60.17 \pm 6.56$ & $\begin{array}{l}\text { Ischemic stroke or } \\
\text { hemorrhage }\end{array}$ & MMSE & $2 / 4$ & $\begin{array}{l}\text { Electropuncture+ } \\
\text { control treatment }\end{array}$ & $\begin{array}{l}\text { Conventional } \\
\text { treatment }+ \\
\text { Oxiracetam }+ \\
\text { hyperbaric } \\
\text { oxygen therapy }\end{array}$ & $\begin{array}{l}\text { FNACCVD confirmed } \\
\text { by head CT or MRI }\end{array}$ & MMSE \\
\hline Jia and Lv (2018) & 40 & 39 & $58.33 \pm 11.13$ & $57.45 \pm 12.37$ & $\begin{array}{l}\text { Ischemic stroke or } \\
\text { hemorrhage }\end{array}$ & MMSE & 4 & $\begin{array}{l}\text { Acupuncture+ } \\
\text { control treatment }\end{array}$ & $\begin{array}{l}\text { Conventional } \\
\text { treatment }+ \\
\text { Huoxuetongluo } \\
\text { decoction }\end{array}$ & $\begin{array}{l}\text { FNACCVD confirmed } \\
\text { by head CT or MRI }\end{array}$ & CCSE \\
\hline Liu (2017) & 32 & 32 & $56.9 \pm 10.3$ & $56.4 \pm 10.1$ & $\begin{array}{l}\text { Ischemic stroke or } \\
\text { hemorrhage }\end{array}$ & MMSE & 2 & $\begin{array}{l}\text { Electropuncture+ } \\
\text { control treatment }\end{array}$ & $\begin{array}{l}\text { Conventional } \\
\text { treatment }+ \\
\text { rehabilitation }\end{array}$ & $\begin{array}{l}\text { FNACCVD confirmed } \\
\text { by head CT or MRI }\end{array}$ & Not shown \\
\hline Liu et al. (2015b) & 19 & 16 & $52.42 \pm 7.62$ & $51.06 \pm 11.62$ & $\begin{array}{l}\text { Ischemic stroke or } \\
\text { hemorrhage }\end{array}$ & MMSE and MoCA & 4 & $\begin{array}{l}\text { Electropuncture+ } \\
\text { control treatment }\end{array}$ & $\begin{array}{l}\text { Conventional } \\
\text { treatment }+ \\
\text { rehabilitation }\end{array}$ & $\begin{array}{l}\text { CECS, Chinese expert } \\
\text { consensus standards }\end{array}$ & MMSE \\
\hline Sun et al. (2013) & 60 & 60 & $62.50 \pm 4.52$ & $63.01 \pm 4.67$ & Ischemic stroke & MMSE & 4 & $\begin{array}{l}\text { Electropuncture+ } \\
\text { control treatment }\end{array}$ & $\begin{array}{l}\text { Conventional } \\
\text { treatment }+ \\
\text { rehabilitation }+ \\
\text { nimodipine }\end{array}$ & $\begin{array}{l}\text { FNACCVD confirmed } \\
\text { by head CT or MRI }\end{array}$ & MMSE \\
\hline $\begin{array}{l}\text { Liu and Feng } \\
\text { (2013) }\end{array}$ & 25 & 25 & \multicolumn{2}{|c|}{$53.40 \pm 8.48$} & $\begin{array}{l}\text { Ischemic stroke or } \\
\text { hemorrhage }\end{array}$ & MMSE & 4 & $\begin{array}{l}\text { Electropuncture+ } \\
\text { control treatment }\end{array}$ & $\begin{array}{l}\text { Conventional } \\
\text { treatment }+ \\
\text { rehabilitation }\end{array}$ & $\begin{array}{l}\text { FNACCVD confirmed } \\
\text { by head CT or MRI }\end{array}$ & DSM-IV-R \\
\hline Wang et al. (2014) & 30 & 30 & \multicolumn{2}{|c|}{$45 \sim 80$} & Ischemic stroke & MoCA & 12 & $\begin{array}{l}\text { Acupuncture+ } \\
\text { control treatment }\end{array}$ & $\begin{array}{l}\text { Conventional } \\
\text { treatment }+ \\
\text { nimodipine }\end{array}$ & $\begin{array}{l}\text { FNACCVD confirmed } \\
\text { by head CT or MRI }\end{array}$ & Not shown \\
\hline $\begin{array}{l}\text { Li and Zhang } \\
\text { (2008) }\end{array}$ & 20 & 20 & \multicolumn{2}{|c|}{$58 \sim 76$} & Ischemic stroke & MMSE & 4 & $\begin{array}{l}\text { Electropuncture+ } \\
\text { control treatment }\end{array}$ & $\begin{array}{l}\text { Conventional } \\
\text { treatment }+ \\
\text { rehabilitation }\end{array}$ & $\begin{array}{l}\text { FNACCVD confirmed } \\
\text { by head CT }\end{array}$ & Not shown \\
\hline
\end{tabular}

MMSE, Mini-Mental State Examination; MoCA, Montreal Cognitive Assessment; FNACCVD, Fourth National Academic Conference of Cerebral Vascular Diseases; CT, computed tomography; MRI, magnetic resonance imaging; DSMIV,Diagnostic and Statistical Manual of Mental Disorders(the fourth edition); DSM-IV-R, DSM-IV-Revised edition; CECS, Chinese expert consensus standards, proposed in 2005 for the prevention and treatment of cognitive dysfunction; CCSE, Cognitive Capacity Screening Examination; CECVCl, Chinese Expert consensus on vascular cognitive impairment 2007. 
A

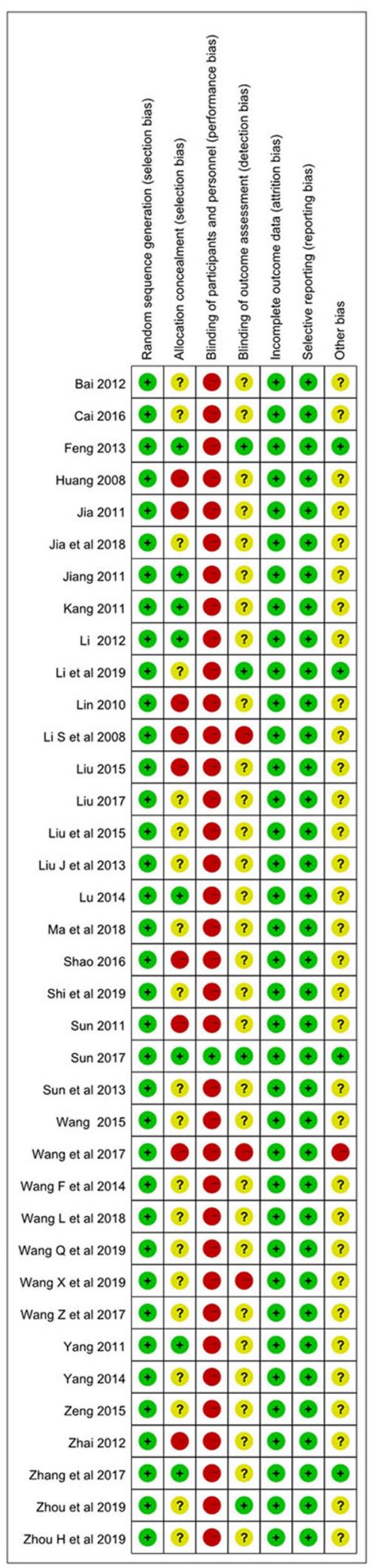

B

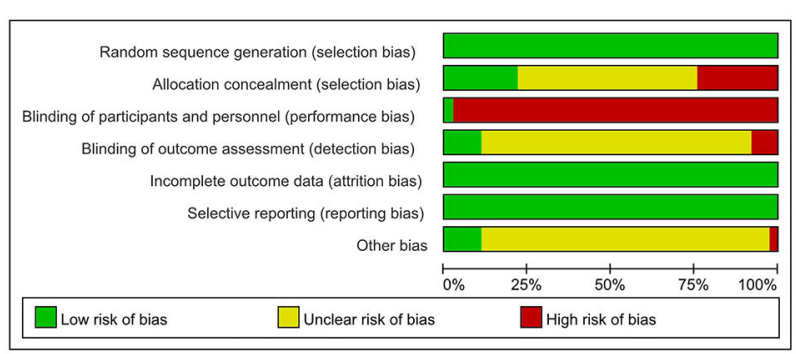

FIGURE 2 | (A) Assessment of risk of bias with selected studies. (B). Risk of bias graph and summary. 
TABLE 2 | Quality assessment of studies.

\begin{tabular}{|c|c|c|c|c|c|c|c|}
\hline References & $\begin{array}{l}\text { Adequate } \\
\text { sequence } \\
\text { generation }\end{array}$ & $\begin{array}{l}\text { Allocation } \\
\text { concealment }\end{array}$ & $\begin{array}{l}\text { Blinding of } \\
\text { participation and } \\
\text { personnel }\end{array}$ & $\begin{array}{l}\text { Blinding of } \\
\text { outcome } \\
\text { assessment }\end{array}$ & $\begin{array}{l}\text { Incomplete } \\
\text { outcome data } \\
\text { addressed }\end{array}$ & $\begin{array}{l}\text { Selective outcome } \\
\text { reporting avoided }\end{array}$ & $\begin{array}{l}\text { Other sources of } \\
\text { bias }\end{array}$ \\
\hline Sun (2017) & Yes & Yes & Yes & Yes & Yes & Yes & Unclear \\
\hline Shao (2016) & Yes & No & No & Unclear & Yes & Yes & Unclear \\
\hline Liu et al. (2015a) & Yes & No & No & Unclear & Yes & Yes & Unclear \\
\hline Cai et al. (2016) & Yes & Unclear & No & Unclear & Yes & Yes & Unclear \\
\hline Zeng et al. (2015) & Yes & Unclear & No & Unclear & Yes & Yes & Unclear \\
\hline Wang (2014) & Yes & Unclear & No & Unclear & Yes & Yes & Unclear \\
\hline Lu (2014) & Yes & Yes & No & Unclear & Yes & Yes & Unclear \\
\hline Zhai (2012) & Yes & No & No & Unclear & Yes & Yes & Unclear \\
\hline Bai et al. (2012) & Yes & Unclear & No & Unclear & Yes & Yes & Unclear \\
\hline Li et al. (2012) & Yes & Yes & No & Unclear & Yes & Yes & Unclear \\
\hline Yang (2011) & Yes & Yes & No & Unclear & Yes & Yes & Unclear \\
\hline $\begin{array}{l}\text { Sun and Wu } \\
\text { (2011) }\end{array}$ & Yes & No & No & Unclear & Yes & Yes & Unclear \\
\hline Kang (2011) & Yes & Yes & No & Unclear & Yes & Yes & Unclear \\
\hline Jiang (2011) & Yes & Yes & No & Unclear & Yes & Yes & Unclear \\
\hline $\begin{array}{l}\text { Jia and Meng } \\
(2011)\end{array}$ & Yes & No & No & Unclear & Yes & Yes & Unclear \\
\hline Lin et al. (2010) & Yes & No & No & Unclear & Yes & Yes & Unclear \\
\hline Huang et al. (2008) & Yes & No & No & Unclear & Yes & Yes & Unclear \\
\hline Shi and Wei (2019) & Yes & Unclear & No & Unclear & Yes & Yes & Unclear \\
\hline $\begin{array}{l}\text { Zhou J. et al. } \\
\text { (2019) }\end{array}$ & Yes & Unclear & No & Unclear & Yes & Yes & Unclear \\
\hline Feng (2013) & Yes & Yes & No & Yes & Yes & Yes & No \\
\hline Wang et al. (2019) & Yes & Unclear & No & Unclear & Yes & Yes & Unclear \\
\hline Li et al. (2019) & Yes & Unclear & No & Yes & Yes & Yes & No \\
\hline Zhang et al. (2017) & Yes & Yes & No & Unclear & Yes & Yes & No \\
\hline $\begin{array}{l}\text { Wang H. et al. } \\
(2017)\end{array}$ & Yes & No & No & No & Yes & Yes & Unclear \\
\hline $\begin{array}{l}\text { Zhou H. et al. } \\
\text { (2019) }\end{array}$ & Yes & Unclear & No & Unclear & Yes & Yes & Unclear \\
\hline $\begin{array}{l}\text { Wang Z. et al. } \\
(2017)\end{array}$ & Yes & Unclear & No & Unclear & Yes & Yes & Unclear \\
\hline Wang et al. (2019) & Yes & Unclear & No & Unclear & Yes & Yes & Unclear \\
\hline $\begin{array}{l}\text { Wang and Li } \\
\text { (2018) }\end{array}$ & Yes & Unclear & No & Unclear & Yes & Yes & Unclear \\
\hline Yang (2014) & Yes & Unclear & No & Unclear & Yes & Yes & Unclear \\
\hline Ma et al. (2018) & Yes & Unclear & No & Unclear & Yes & Yes & Unclear \\
\hline Jia and Lv (2018) & Yes & Unclear & No & Unclear & Yes & Yes & Unclear \\
\hline Liu (2017) & Yes & Unclear & No & Unclear & Yes & Yes & Unclear \\
\hline Liu et al. (2015b) & Yes & Unclear & No & Unclear & Yes & Yes & Unclear \\
\hline Sun et al. (2013) & Yes & Unclear & No & Unclear & Yes & Yes & Unclear \\
\hline $\begin{array}{l}\text { Liu and Feng } \\
\text { (2013) }\end{array}$ & Yes & Unclear & No & Unclear & Yes & Yes & Unclear \\
\hline Wang et al. (2014) & Yes & Unclear & No & Unclear & Yes & Yes & Unclear \\
\hline $\begin{array}{l}\text { Li and Zhang } \\
\text { (2008) }\end{array}$ & Yes & No & No & No & Yes & Yes & Unclear \\
\hline
\end{tabular}

\section{DISCUSSION}

Our findings showed that acupuncture or electroacupuncture therapy is effective in improving the cognitive impairment of post-stroke patients by assessing with MMSE and MoCA. The gain of the mean difference is 2.88 for MMSE (CI $[2.09,3.66]$ ), which is significant in clinical treatment (Andrews et al., 2019). The gain of the mean difference is 2.66 for MoCA (CI [1.95, 3.37]), which is also significant in clinical treatment (Wong et al., 2017).

In this study, patients in the control group were treated with conventional treatment in all 37 trials, patients had 


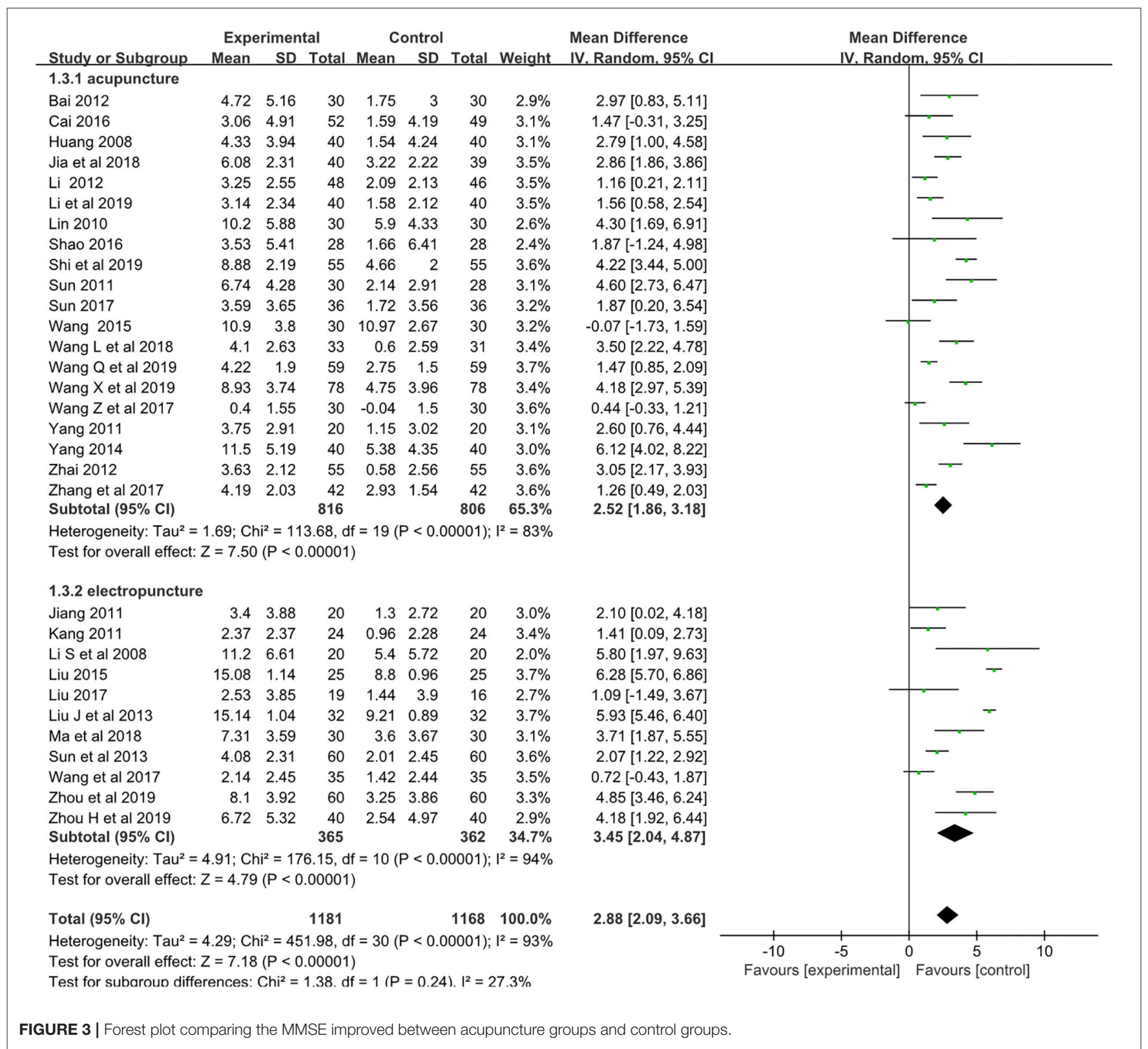

conventional rehabilitation done in the control groups in 23 trials, and patients had medicine in control groups in 17 trials. Patients in experiment groups combined the acupuncture or electroacupuncture and conventional rehabilitation or medicine used the same as in control groups in all trials. The merged results showed that synergistic effects of acupuncture or electroacupuncture therapy is clinically significant in improving PSCI, and there were no adverse events/incidents reported in those studies.

There was some inconsistent information in the included studies. The patients were all with ischemic stroke in 10 studies; the other 22 studies included patients with hemorrhage or ischemic stroke, and 4 studies only included post-stroke patients. In the 37 studies, 23 studies indicated that acupuncture treatment was within 6 months from stroke onset, 5 studies was under 1 year, 2 studies was under 14/36 months, and the other 7 studies did not report the accurate time. In this meta-analysis, 24 studies focused on the effects of acupuncture combined with conventional rehabilitation treatment, and the other 13 studies analyzed the effectiveness of acupuncture combined with medicine (Aricept, Xingnaojing, Nimodipine, Piracetam, etc.). Fourteen studies used electroacupuncture, and the other 23 studies used traditional manual acupuncture. The intervention period varied across studies from 2 to 12 weeks.

There were obvious heterogeneities of these articles, so the random effects model was used in this study. Subgroup analysis between acupuncture group and electropuncture did not significantly reduce heterogeneity in this study. This may 


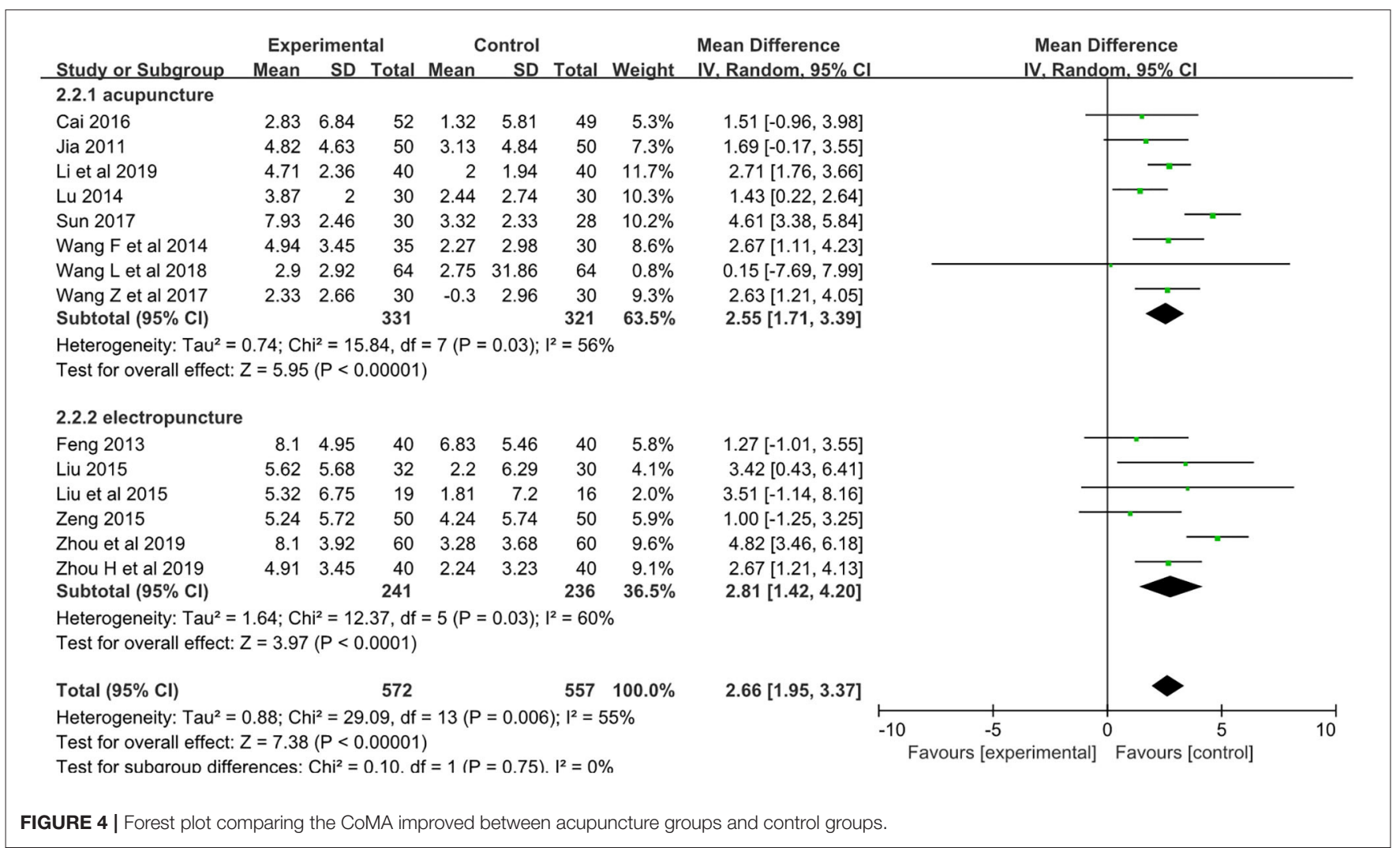

be caused by the unbalance of acupoints selection, the different treatment period, and the therapist's technical ability.

In the theoretical system of acupuncture, the Du Meridian is important for the cognitive brain function (Zhou et al., 2013; Liu et al., 2014), and acupoints "Baihui" and "Shenting" belong to the Du Meridian. Baihui and Shenting are both located in the head. To Chinese traditional medicine theoretical system, acupuncture Baihui and Shenting can lift the spirit, clear the mind, and promote resuscitation. In these 37 studies, 26 studies acupuncture the acupoints including "Baihui," and 19 studies involved the acupoint "Shenting" for the treatment of mental and emotional illness. Other acupoints, such as Feishu, Xinshu, Ganshu, Shenshu, and Pishu were shown involving the cognitive function in more than three studies. Other acupuncture points involved were Huiyin, Yintang, Neiguan, Yanglingquan, Taixi, Zulinqi, Sishencong, Fengchi, Fengfu, Gongxue, Yiming, Guanyuan, Taichong, Shenshu, Benshen, Hegu, Taichong, Fengshi, Quchi, Zusanli, Sanyingjiao, Xuehai, Renzhong, Shenmen, etc.

Acupuncture improves cognitive function and depressive disorder, because acupuncture on stroke patients can improve neurological function (Chen et al., 2018; Hung et al., 2019). Animal studies showed that acupuncture with Baihui may have a neuroprotective effect via decreasing MMP-9 expression or improving the endothelial nitric oxide synthase (eNOS)mediated perfusion (Dong et al., 2009; Kim et al., 2013).

$\mathrm{H}$ Jiang et al.'s and J Liang et al.'s studies showed that acupuncture was associated with the potential of DNA methylation and histone modifications of brain-derived neurotropic factor in epigenetic mechanism, which can produce antidepressant effect in rats (Liang et al., 2012; Jiang et al., 2018). $\mathrm{F}$ Taya et al. showed that acupuncture may increase cerebral collateral circulation, promoting repair of the lesion (Taya et al., 2015). P.Y. Sun et al. showed that acupuncture repairs hippocampal neuronal damage, which is probably related to the contents of hippocampal monoamine neurotransmitters (NE, 5-HT and DA) (Sun et al., 2019). Other studies showed that electropuncture can improve cognitive function via synaptic plasticity by attenuating pathological lesions and increasing the density of dendritic spines and number of CA1 synapses in rats (Lin et al., 2016; Liu et al., 2017; Wen et al., 2018).

The selection criteria for the assessment of cognitive function were the Mini-Mental State Examination (MMSE) and the Montreal Cognitive Assessment (MoCA). MMSE is an effective tool that can be used to systematically and thoroughly assess mental status, which was validated and extensively used from 1975 (Foreman et al., 1996). MoCA is a widely used screening assessment for detecting cognitive impairment since 1996, which was validated in the setting of mild cognitive impairment (Nasreddine et al., 2005). There are other internationally recognized examinations of cognitive impairment including NCSE, NIHSS, LOTCA, HDS, and cognitive potential 300, but the most commonly used indicators are MMSE and MoCA. We restricted the inclusion criteria to a consistent standard of outcome assessing with MMSE or MoCA, so the number of RCTs included in this study was not so many 


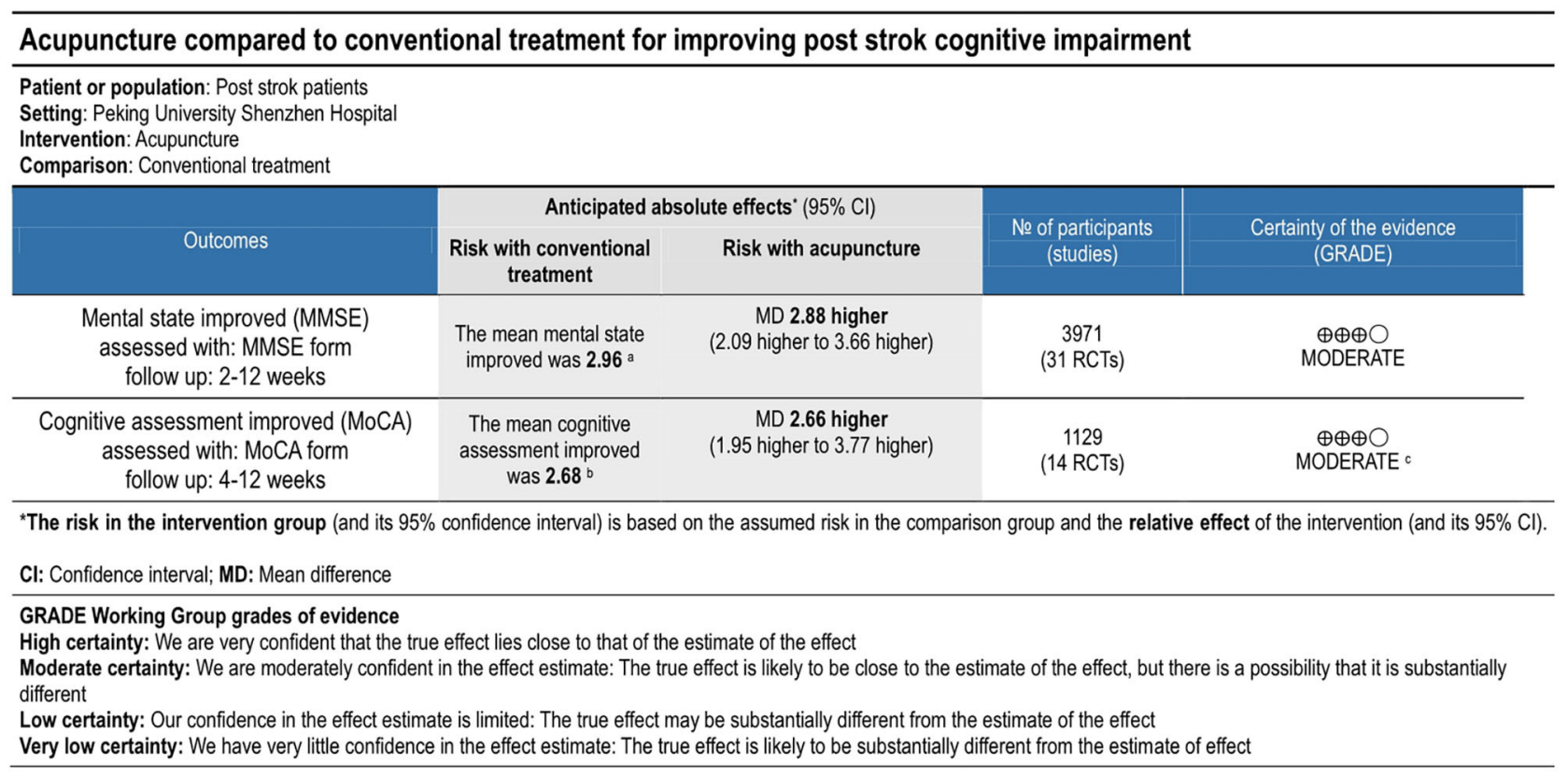

\section{Explanations}

a. After conventional treatment, the MMSE score was improved by 2.97 than before.

b. After conventional treatment, the MoCA score was improved by 2.68 than before.

c. Many of the RCTs were low quality with an inadequate level of blinding because blindings in the acupuncture therapy were difficult for the therapists and patients.

FIGURE 5 | Evidence profile.

(only 37), but the results of meta-analyses were more clear and definite, and the quality of evidence was assessed to be moderate.

About the limitation, firstly, all studies were done in China, although the Cai et al. (2016) study was published in the English language. There might have been additional reports using non-Chinese or non-English languages that were not included which may limit the results of the study. Secondly, many of the trials were of low quality with an inadequate level of blinding; although blinding in the acupuncture therapy is difficult for the therapists and patients, blinding the assessor is necessary.

Despite these limitations, conclusions can be drawn from the results of our study.

\section{CONCLUSIONS}

Acupuncture therapy has positive synergistic effects in improving PSCI, but more rigorous design studies with large-scale sham are needed to determine the longevity of acupuncture effects.

\section{REFERENCES}

NIH consensus conference (1998). acupuncture. JAMA 280, 1518-1524. doi: $10.1001 /$ jama.280.17.1518

\section{DATA AVAILABILITY STATEMENT}

The original contributions presented in the study are included in the article/supplementary materials, further inquiries can be directed to the corresponding author/s.

\section{AUTHOR CONTRIBUTIONS}

LZ, QW, and XL: conceptualization and writing, review, and editing. LZ, YW, and JQ: data curation and methodology. LZ and XL: funding acquisition. QW and XL: supervision. LZ: writing the original draft. All authors contributed to the article and approved the submitted version.

\section{FUNDING}

LZ and YW received the foundation of the Sanming Project of Medicine in Shenzhen (SZSM201612065 and SZSM201610039); $\mathrm{LZ}$ also received the foundation of Clinical research of Shenzhen Municipal Health Commission (SZLY2017006).

Andrews, J., Desai, U., Kirson, N., Zichlin, M., Ball, D., and Matthews, B. (2019). Disease severity and minimal clinically important differences in clinical outcome assessments for Alzheimer's disease clinical trials. Alzheimer's Dementia 5, 354-363. doi: 10.1016/j.trci.2019.06.005 
Bai, J., Li, B., and Wang, Q. (2012). Therapeutic observation on cluster needling at scalp acupoints plus cognitio training for post-stroke cognitive impairment. Shanghai J. Acupunct. Moxibustion 10, 711-713. doi: 10.3969/j.issn.1005-0957.2012.10.711

Berrol, S. (1990). Issues in cognitive rehabilitation. Arch. Neurol. 47, 219-220. doi: 10.1001/archneur.1990.00530020127025

Cai, J., Yang, S. L., Tao, J., Huang, J., Li, Y. Y., Ye, H. C., et al. (2016). Clinical efficacy of acupuncture treatment in combination with rehacom cognitive training for improving cognitive function in stroke: a $2 \times 2$ factorial design randomized controlled trial. J. Am. Med. Dir. Assoc. 17, 1114-1122. doi: 10.1016/j.jamda.2016.07.021

Chen, A., Gao, Y., Wang, G., Li, J., and Shen, W. (2018). Effect of early acupuncture intervention on post-stroke depression: a randomized controlled trial. Zhongguo Zhen Jiu 38, 1141-1144. doi: 10.13703/j.0255-2930.2018.11.001

Choi, J., and Twamley, E. W. (2013). Cognitive rehabilitation therapies for Alzheimer's disease: a review of methods to improve treatment engagement and self-efficacy. Neuropsychol. Rev. 23, 48-62. doi: 10.1007/s11065-013-9227-4

Dong, H., Fan, Y. H., Zhang, W., Wang, Q., Yang, Q. Z., and Xiong, L. Z. (2009). Repeated electroacupuncture preconditioning attenuates matrix metalloproteinase- 9 expression and activity after focal cerebral ischemia in rats. Neurol. Res. 31, 853-858. doi: 10.1179/174313209X393960

Du, S. J., Su, X., Feng, W. F., Chen, X. J., Xu, C. Y., Feng, S. W., et al. (2018). Observation on the therapeutic effect of TiaoShenJiangPi acupuncture combined with tonepezil on cognitive impairment after stroke. J. Pract. Tradit. Chinese Med. 7, 810-811. doi: 10.3969/j.issn.1004-2814.2018.07.047

Feng, X. (2013). The Studies of clinical Observation and Mechanism in Treating the Cognitive Impairment After Stroke by Electroacupuncture at Shenting and Baidui. (M.D) Fujian University of Traditional Chinese Medicine, Fuzhou, China.

Foreman, M. D., Fletcher, K., Mion, L. C., and Simon, L. (1996). Assessing cognitive function. Geriat. Nurs. 17, 228-232. doi: 10.1016/S0197-4572(96)80210-2

Guo, R. Y., Liu, L. A., and Ma, X. W. (2007). Long-term effect of acupuncture on quality of life in patients with early stage of stroke. Zhongguo Zhong Xi Yi Jie He Za Zhi 7, 708-710. doi: 10.3321/j.issn:1003-5370.2007.08.012

Hu, H. H., Chung, C., Liu, T. J., Chen, R. C., Chen, C. H., Chou, P., et al. (1993). A randomized controlled trial on the treatment for acute partial ischemic stroke with acupuncture. Neuroepidemiology 12, 106-113. doi: 10.1159/000110308

Huang, F., Liu, Y., Zhou, F., Yao, G., and He, Q. (2008). Effect of acupuncture on vascular cognitive impairment after cerebral infarction. Guangdong Med. J. 11, 1918-1920. doi: 10.3969/j.issn.1001-9448.2008.11.071

Hung, C. Y., Wu, X. Y., Chung, V. C., Tang, E. C., Wu, J. C., and Lau, A. Y. (2019). Overview of systematic reviews with meta-analyses on acupuncture in poststroke cognitive impairment and depression management. Integrat. Med. Res. 8, 145-159. doi: 10.1016/j.imr.2019.05.001

Jia, J. P. (2004). Attention should be paid to the establishment of diagnostic criteria and clinical research of vascular cognitive impairment. Chinese J. Cerebrovas. Dis. 1, 14-17. doi: 10.3969/j.issn.1672-5921.2004.01.004

Jia, X., and Meng, I. (2011). Observations on the efficacy of lower point selection for upper disease in treating cognitive impairment after acute cerebral infarction. Shanghai J. Acupunct. Moxibustion 30, 589-590. doi: 10.3969/j.issn.1005-0957.2011.09.589

Jia, Y., and Lv, X. (2018). Clinical study of acupuncture combined with Huoxue Tonglno Decoction in the treatment of post-stroke cognitive impairmen. China Med. Herald 4, 113-116.

Jiang, H., Zhang, X., Lu, J., Meng, H., Sun, Y., Yang, X., et al. (2018). Antidepressant-like effects of acupuncture insights from DNA methylation and histone modifications of brain-derived neurotrophic factor. Front. Psychiatry 9:102. doi: 10.3389/fpsyt.2018.00102

Jiang, Y. (2011). Electroacupuncture DU20 and DU24 treatment on cognitive impairment. (M.S) Fujian University of Traditional Chinese Medicine, Fuzhou, China.

Johansson, K., Lindgren, I., Widner, H., Wiklund, I., and Johansson, B. B. (1993). Can sensory stimulation improve the functional outcome in stroke patients? Neurology 43, 2189-2192. doi: 10.1212/WNL.43.11.2189

Kang, J. (2011). Clinical study of effect of electroacupuncture on GV20 and EXHN1 on stroke patients with cognitive impairment. M.S. (Fujian University of Traditional Chinese Medicine).
Kim, J. H., Choi, K. H., Jang, Y. J., Bae, S. S., Shin, B. C., Choi, B. T., et al. (2013). Electroacupuncture acutely improves cerebral blood flow and attenuates moderate ischemic injury via an endothelial mechanism in mice. PLoS ONE 8:e56736. doi: 10.1371/journal.pone.0056736

Lee, J. D., Chon, J. S., Jeong, H. K., Kim, H., Yun, M., Kim, D. Y., Kim, D. I., et al. (2003). The cerebrovascular response to traditional acupuncture after stroke. Neuroradiology 45, 780-784. doi: 10.1007/s00234-003-1080-3

Li, L., Xiao, P., Chen, Q., and Tang, L. (2019). Effects of acupuncture combined with donepezil on cognitive impairment after stroke in elderly patients. Chinese J. Prevent. Control Chronic Non-Commun Dis. 27, 617-620. doi: 10.16386/j.cjpccd.issn.1004-6194.2019.08.015

Li, S. M., and Zhang, Z. X. (2008). The effect of acupuncture on the patients with cerebral infarction. Zhejiang Chinese Med. Univ. xue bao 24, 514-515. doi: 10.3969/j.issn.1005-5509.2008.04.051

Li, W., Cheng, Y. H., and Yu, X. G. (2012). Observation on therapeutic effect of acupuncture combined with medicine on mild cognition disorders in patients with post-stroke. Chinese Acupunct. Moxibust. 32, 3-7. doi: $10.13703 / \mathrm{j} .0255-2930.2012 .01 .005$

Liang, J., Lu, J., Cui, S. F., Wang, J. R., and Tu, Y. (2012). Effect of acupuncture on expression ofbrain-derived neurotrophic factor gene and protein in frontal cortex and hip-pocampus of depress rats. Zhen Ci Yan Jiu 37, 20-24. doi: $10.13702 / j .1000-0607.2012 .01 .009$

Lin, H., Ding, X., and Fu, B. (2010). Effects of acupuncture combined with medicine on cognitive impairment of post-stroke patients. Modern J. Integrat. Tradit. Chinese West. Med. 1, 36-37. doi: 10.3969/j.issn.1008-8849.2010.01.017

Lin, R., Wu, Y., Tao, J., Chen, B., Chen, J., Zhao, C., et al. (2016). Electroacupuncture improves cognitive function through Rho GTPases and enhances dendriticspine plasticity in rats with cerebral ischemia-reperfusion. Mol. Med. Rep. 13, 2655-2660. doi: 10.3892/mmr.2016.4870

Liu, F., Li, Z. M., Jiang, Y. J., and Chen, L. D. (2014). A meta-analysis of acupuncture use in the treatment of cognitive impairment after stroke. J. Alter. Complement. Med. 20, 535-544. doi: 10.1089/acm.2013.0364

Liu, J., and Feng, X. (2013). Clinical observation of treating cognitive impairment after stroke by electroacupuncture at Baihui and Shenfing with cognitive rehabilitation training. J. Chinese Med. 4, 608-610. doi: 10.16368/j.issn.1674-8999.2013.04.020

Liu, L., Li, H., Chen, Z., Xu, J., and Lu, H. (2015b). Effects of electroacupuncture on head shen-acupoints on cognitive impairment after stroke. Chinese J. Rehabil. Theory Pract. 21, 575-578. doi: 10.3969/j.issn.1006-9771.2015.05.018

Liu, L., Li, H., and Xu, J. (2015a). Clinical study of electroacupuncture on memory dysfunction after stroke. J. Emerg. Tradit. Chinese Med. 5, 775-777. doi: 10.3969/j.issn.1004-745X.2015.05.008

Liu, R. (2017). Clinical study of electric acupuncture Shenting and Baihui point on mild cognitive impairment after stroke. Clin. Res. Pract. 29, 101-102. doi: 10.19347/j.cnki.2096-1413.201729049

Liu, W., Wu, J., Huang, J., Zhuo, P., Lin, Y., Wang, L., et al. (2017). Electroacupuncture regulateshippocampal synapitc plasticity via miR-134mediated LIMK1 function in ratswith ischemic stroke. Neural. Plast. 2017:9545646. doi: 10.1155/2017/9545646

Lu, Z. (2014). Clincal research of post-stroke vascular cognitive impairment treatent with needling back-shu point. M.S. (Guangzhou University of Chinese Medicine).

Ma, Y., Han, Z., Wu, Y., Liu, P., and Peng, S. (2018). Effect of hyperharic oxygen combine with electroacupuncture at Siguan acupoint on early cognitive impairment in stroke patients. Surg. Res. N. Techniq. 1, 38-40. doi: 10.3969/j.issn.2095-378X.2018.01.011

Merriman, N. A., Sexton, E., McCabe, G., Walsh, M. E., Rohde, D., Gorman, A., et al. (2019). Addressing cognitive impairment following stroke: systematic review and meta-analysis of non-randomised controlled studies of psychological interventions. BMJ Open 9:e024429. doi: 10.1136/bmjopen-2018-024429

Nakling, A. E., Aarsland, D., Næss, H., Wollschlaeger, D., Fladby, T., Hofstad, H., et al. (2017). Cognitive deficits in chronic stroke patients: neuropsychological assessment. depression, and self-reports. Dement. Geriatr. Cogn. Dis. Extra 7, 283-296. doi: 10.1159/000478851

Nasreddine, Z. S., Phillips, N. A., Bédirian, V., Charbonneau, S., Whitehead, V., Collin, I., et al. (2005). The Montreal Cognitive Assessment, MoCA: a brief 
screening tool for mild cognitive impairment. J. Am. Geriatr. Soc. 53, 695-699. doi: 10.1111/j.1532-5415.2005.53221.x

Nys, G. M., van Zandvoot, M. J., van der Worp, H. B., de Kort, P. L., Jansen, B. P., Kappelle, L. J., et al. (2006). Early cognitive impairment predicts long-term depressive symptoms and qulity of life after stroke. J. Neurol. Sci. 247, 149-156. doi: 10.1016/j.jns.2006.04.005

Pasi, M., Poggesi, A., Salvadori, E., and Pantoni, L. (2012). Post-stroke dementia and cognitive impairment. Front. Neurol. Neurosci. 30, 65-69. doi: $10.1159 / 000333412$

Patel, M., Coshall, C., Rudd, A. G., and Wolfe, C. D. (2003). Natural history of cognitive impairment after stroke and factors associated with its recovery. Clin. Rehabil. 17, 158-166. doi: 10.1191/0269215503cr596oa

Shao, D. (2016). The effect of scalp acupuncture and neck acupuncture and body acupuncture on the treatments of post-stroke cognitive impairment. Chinese J. Geriatric Care 3, 17-19. doi: 10.3969/j.issn.1672-4860.2016.03.007

Shi, C., and Wei, L. (2019). Effect of Xingnaokaiqiao acupuncture on hemiplegia after stroke. Henan Med. Res. 13, 2433-2434. doi: 10.3969/j.issn.1004-437X.2019.13.066

Song, S., Zhao, J., Tian, J., Wang, Z., Yang, L., and Xiu, X. (2013). The clinical curative efect of acupuncture combined with medicine on stroke patients with cognitive impairment. J. Emerg. Tradit. Chinese Med. 11, 1859-1860.

Sun, P. Y., Cai, R. L., Li, P. F., Zhu, Y., Wang, T., Wu, J., et al. (2019). Protective effects on hippocampal neurons and the influence on hippocampal monoamine neurotransmitters with acupuncture for promoting the circulation of the governor vessel and regulating the mental state in rats with post-stroke depression. Zhongguo Zhen Jiu 39, 741-747. doi: 10.13703/j.0255-2930.2019.07.017

Sun, S. (2017). The clinical study of the treatment in patients with moderate and severe post-stroke cognitive impairment by acupuncture at RN 1. M.S. (Fujian University of Traditional Chinese Medicine).

Sun, S. C., Zhao, J. W., Tian, J. B., Wang, Z. Y., Yang, L. J., and Liu, X. F. (2013). The clinical curative effect of acupuncture combined with medicine on stroke patients with cognitive impairment. J. Emerg. Tradit. Chinese Med. 22, 1859-1860.

Sun, Y., and Wu, W. (2011). The effect of scalp acupuncture of 36 cases of cognitive dysfunction after ischemic stroke. (translation from Chinese by author). J. Clin. Acupunct. Moxibust. 9, 11-13. doi: 10.3969/j.issn.1005-0779.2011.09.004

Tatemichi, T. K., Desmond, D. W., Stern, Y., Paik, M., Sano, M., and Bagiella, E. (1994). Cognitive impairment after stroke: frequency, patterns, and relationship to functional abilities. J. Neurol. Neurosurg. Psychiatr 57, 202-207. doi: 10.1136/jnnp.57.2.202

Taya, F., Sun, Y., Babiloni, F., Thakor, N., and Bezerianos, A. (2015). Brain enhancement through cognitive training: a new insight from brain connectome. Front. Syst. Neurosci. 9:44. doi: 10.3389/fnsys.2015.00044

Wang, F., Liang, H., Chen, S., Huang, J., and Lin, Q. Y. (2014). Magnetic resonance spectroscopy of acupuncture regulating brain tissue metabolism in treatment of mild cognitive impairment after stroke. (translation from Chinese by author). J. Emerg. Tradit. Chinese Med. 10, 1928-1930. doi: 10.3969/j.issn.1004-745X.2014.10.072

Wang, H., Feng, X., and Chen, Z. (2017). Clinical efficacy of electro-acupuncturing on Baihui and Zusanli points plus rehabilitation training on post-stroke cognitive impairment. Clin. J. Chinese Med. 5, 67-70.

Wang, J. (2017). The Literature EvaIuation and Clinical Research of Treating PSCl With Acupuncture and Moxibustion. (M.D.), Guangzhou University of Chinese Medicine, Guangzhou, China.

Wang, L., and Li, W. (2018). Effect of Xingnao Kaiqiao acupuncture on clinical efficacy of patients with mild cognitive impairment after stroke and its mechanism. Chinese J. Integrat. Tradit. West. Med. Intens. Critical Care 3, 260-263. doi: 10.3969/j.issn.1008-9691.2018. 03.010

Wang, Q. (2014). Analysis of cognitive impairment after stroke treated by acupuncture combined with drugs. Chinese J. Moderm Drug Appl. 8:239. doi: 10.14164/j.cnki.cn11-5581/r.2014.14.199

Wang, Q., Dong, J., and Sun, L. (2019). Effect of acupuncture combined with atorvastatin on hemorheology and cognitive status in elderly patients with mild cognitive impairment after ischemic stroke(translation from Chinese by author). Chinese J. Gerontol. 21, 5180-5183. doi: 10.3969/j.issn.1005-9202.2019.21.012

Wang, X. (2019). Effects of tongluo fuzheng decoction combined with acupuncture on limb function and cognitive function in elderly patients with post-stroke hemiplegia. Nei Mongol J. Tradit. Chinese Med. 2, 30-31. doi: 10.3969/j.issn.1006-0979.2019.02.022

Wang, Z., Zhang, H., Zhang, Y., and Gu, Z. (2017). A clinical study on the treatment of vascular mild cognitive impairment with kidneytonifying turbid acupuncture. Chinese J. Woman Child Health Res. 28:127. doi: 10.3969/j.issn.1674-7860.2017.05.035

Wen, T., Zhang, X., Liang, S., Li, Z., Xing, X., Liu, W., et al. (2018). Electroacupuncture amelio-rates cognitive impairment and spontaneous lowfrequency brain activity inrats with ischemic stroke. J. Stroke Cerebrovas. Dis. 27, 2596-2605. doi: 10.1016/j.jstrokecerebrovasdis.2018.05.021

Wong, G. K. C., Mak, J. S. Y., Wong, A., Zheng, V. Z. Y., Poon, W. S., Abrigo, J., et al. (2017). Minimum clinically important difference of montreal cognitive assessment in aneurysmal subarachnoid hemorrhage patients. J. Clin. Neurosci. 46, 41-44. doi: 10.1016/j.jocn.2017.08.039

Wu, P., Mills, E., Moher, D., and Seely, D. (2010). Acupuncture in poststroke rehabilitation: a systematic review and meta-analysis of randomized trials. Stroke 41, e171-179. doi: 10.1161/STROKEAHA.109.573576

Yang, H. (2014). Observation on the effect of acupuncture combined with drugs on cognitive impairment after stroke(translation from Chinese by author). Guangming J. Chinese Med. 29, 1680-1681. doi: 10.3969/j.issn.1003-8914.2014.08.051

Yang, J. (2011). The Clinical study on cognitive impairment after stroke by using the treatment of electroacupuncture given at DU20 and GB20. M.S. (Fujian University of Traditional Chinese Medicine).

Zeng, Y., Bao, Y., Zhu, M., Chen, S., and Fang, J. (2015). Mild cognitive impairment of stroke at subacute stage treated with acupuncture: a randomized controlled trial. Chinese Acupunct. Moxibust. 35, 979-982. doi: 10.13703/j.0255-2930.2015.10.001

Zhai, W. Q. (2012). Effect of acupuncture combined with rehabilitation training on cognitive dysfunction after cerebral infarction. Healthy People. 6, 18-19.

Zhang, X., Y. Z., Guo, Z., Liu, J., and Jiao, X. (2017). Effect of acupuncture at cervical Jiaji point and Du Channel Point combined with atorvastatin on hemodynamics in patients with mild cognitive impairment after stroke. $J$. Hunan Normal Univ. 14, 131-134. doi: 10.3969/j.issn.1673-016X.2017.02.041

Zhang, Y. Y., and Wang, L. Y. (2004). Diagnosis and intervention of mild cognitive impairment. Chinese J. Epidemiol. 25, 905-907. doi: 10.3760/j.issn:0254-6450.2004.10.021

Zhou, H., Qing, S., Huang, D., and Huang, C. (2019). Effect of acupuncture combined with perindopril on cognitive function in ischemic stroke patients. (translation from Chinese by author). Chinese Commun. Doct. 4, 129-131. doi: 10.3969/j.issn.1007-614x.2019.04.085

Zhou, J., Zuo, J., Chen, B., and Lu, J. (2019). Effects of electroacupuncture at Baihui (GV20) and Shenting (GV24) on mild cognitive impairment after stroke. World Chinese Med. 2, 486-489. doi: 10.3969/j.issn.1673-7202.2019.02.050

Zhou, L., Zhang, Y. L., Cao, H. J., and Hu, H. (2013). Treating vascular mild cognitive impairment by acupuncture: a systematic review of randomized controlled trials. Chinese J. Integrat. Tradit. West. Med. 33, 1626-1630. doi: 10.7661/CJIM.2013.12.1626

Conflict of Interest: The authors declare that the research was conducted in the absence of any commercial or financial relationships that could be construed as a potential conflict of interest.

Copyright (c) 2020 Zhou, Wang, Qiao, Wang and Luo. This is an open-access article distributed under the terms of the Creative Commons Attribution License (CC BY). The use, distribution or reproduction in other forums is permitted, provided the original author(s) and the copyright owner(s) are credited and that the original publication in this journal is cited, in accordance with accepted academic practice. No use, distribution or reproduction is permitted which does not comply with these terms. 